\title{
Pathology of blast-related brain injury
}

\author{
Jeffery D. Kocsis, PhD; ${ }^{1-2 *}$ Alan Tessler, $\mathbf{M D}^{3}$ \\ ${ }^{1}$ Department of Neurology and Center for Neuroscience and Regeneration Research, Yale University School of \\ Medicine, New Haven, CT; ${ }^{2}$ Rehabilitation Research Center, Department of Veterans Affairs (VA) Connecticut \\ Healthcare System, West Haven, CT; ${ }^{3}$ Philadelphia VA Medical Center, Department of Neurology, Philadelphia, PA
}

\begin{abstract}
Blasts are responsible for about two-thirds of the combat injuries in Operation Iraqi Freedom and Operation Enduring Freedom, which include at least 1,200 traumatic brain injuries. Blasts inflict damage to the brain directly and by causing injuries to other organs, resulting in air emboli, hypoxia, and shock. Direct injuries to the brain result from rapid shifts in air pressure (primary blast injury), from impacts with munitions fragments and other objects propelled by the explosion (secondary blast injury), and from collisions with objects and rapid acceleration of individuals propelled by the explosion (tertiary blast injury). Tertiary injury can occur from a building or other structure collapsing and from an individual being thrown by the blast wind. The pathological consequences of secondary and tertiary blast injuries are very likely to be similar to those of other types of mechanical trauma seen in civilian life. The damage attributable to the specific effects of a blast, however, has received little study, although it has been assumed to include the focal and diffuse lesions characteristic of closed head injuries. Available clinical studies of blast injuries show focal damage similar to that found in other types of closed head injuries but have not determined whether diffuse axonal injury also occurs. In this article, we will try to reach a better understanding of the specific pathology of blast-related brain injury by reviewing the available experimental studies and the autopsy reports of victims of terrorist attacks and military casualties dating back to World War I.
\end{abstract}

Key words: barotrauma, blast injury, blast overpressure, blastrelated brain injury, blast underpressure, central nervous system, closed head injury, improvised explosive devices, neuropathology, traumatic brain injury.

\section{INTRODUCTION}

Improvised explosive devices (IEDs) are being increasingly used in insurgent and terrorist activities. Injuries caused by IEDs result from blast wave-induced changes in atmospheric pressure (primary blast injury), from objects projected from the blasts into people (secondary blast injury), and from people being propelled from the blast (tertiary blast injury) [1-2]. A recent study found that 47 percent of blast-related injuries in Iraq involved the head [3]. Early literature describes a condition in which soldiers coming in contact with powerful explosions were rendered unconscious or dazed when no external injury was noted. Upon regaining consciousness, these soldiers reported severe headache, tinnitus, retrograde and anterograde amnesia, tremors, and hypersensitivity to noise [4-6].

Drs. Gordon Holmes and Fred Mott, the noted physicians with the British Army in World War (WW) I, studied and reported cases of soldiers exposed to blasts who developed neuropsychiatric symptoms. They had difficulty

\footnotetext{
Abbreviations: CNS $=$ central nervous system, CSF $=$ cerebrospinal fluid, IED = improvised explosive device, TBI = traumatic brain injury, WW = World War.

*Address all correspondence to Jeffery D. Kocsis, PhD; Yale University School of Medicine, Neuroscience Research Center (127A), VA CT Healthcare System, West Haven, CT 06516; 203-937-3802; fax: 203-937-3801.

Email: jeffery.kocsis@yale.edu

DOI:10.1682/JRRD.2008.08.0100
} 
distinguishing between physical brain injury and emotional trauma $[4,7]$. This controversy continued into WWII; some physicians felt there was no organic brain injury, while others reported electroencephalography changes similar to those in confirmed closed head injuries [5-6]. While the brain is clearly vulnerable to secondary and tertiary blast injury, an unresolved controversy is whether primary blast injury directly damages the brain. Shear and stress waves from the primary blast may directly damage the brain, thus causing traumatic brain injury (TBI). The pathology of TBI is multifaceted and may include contusion, edema, hemorrhage, and diffuse axonal damage [8]. Moreover, primary blast could cause formation of gas emboli, leading to infarction [9]. In this brief review, we discuss clinical data related to blast injury and studies using animal models to simulate blast injury.

\section{BLAST WAVE-INDUCED CHANGES IN ATMOSPHERIC PRESSURE}

High-energy explosive detonation results in extremely rapid conversion of a solid or liquid into gases and changes in atmospheric pressure [2,10-11]. The gases expand rapidly, forming a pulse of pressure, or "blast overpressure," from the compression in the surrounding air. With continued gas expansion, the pressure drops, creating a relative vacuum referred to as "blast underpressure.” Thus, the blast wind initially moves away from the explosion, but as atmospheric pressure drops below normal, the blast winds are reversed. These extreme pressure differences (blastinduced over- and underpressure) that occur as the blast wave reaches the body result in stress and shear waves. Primary blast injury is thought to result from these blast waveinduced changes in atmospheric pressure, which is a form of barotrauma.

Barotrauma may result from organs and tissues of different densities being accelerated or decelerated at different rates, resulting in damaging stretching and shearing forces. Air-fluid interfaces such as those present in the lungs, bowel, and middle ear are thought to be the most vulnerable regions of the body. The precise pathological effects of the primary blast wave on brain are not clear. However, as we will discuss, some experimental evidence suggests direct injury to the brain. Clearly, the blast wave can propel objects with considerable force and that these objects can then strike an individual, leading to secondary blast injury. Moreover, individuals can be pro- pelled by the blast wind into objects, causing tertiary blast injury. Collision of objects with the head may result in TBI. The mechanical properties (e.g., stiffness) and speed of the object will determine the forces acting on the head.

\section{PATHOLOGY OF BLAST-RELATED BRAIN INJURY}

Whether a blast wave can directly damage the brain and the pathophysiological mechanisms by which it may do so have been the subjects of controversy since at least WWI [12]. Blast overpressure transmitted through the skull may subject the brain and its blood vessels to the same types of tensile and shear strains that occur after conventional closed head injuries and produce similar types of injuries. Also conceivable is that the unique properties of a blast following transmission through the skull may induce specific patterns of damage. In addition, blasts have been postulated to damage the brain through elevations in cerebrospinal fluid (CSF) or venous pressure due to compression of the thorax and abdomen and by propagation of a shock wave through the blood vessels or CSF [13]. These indirect mechanisms might also have specific effects on the brain. It has proven difficult to resolve the patterns and mechanisms of damage specific for the blast itself on the basis of clinical neuropathological studies. First, even when no obvious signs of external injury to the head exist, secondary and tertiary blast injuries can rarely be excluded, and in some cases, the additional possibility of injury from carbon monoxide or other gas inhalation exists. The possibility of toxic gas injury is a particular concern, because the chaotic conditions that accompany military and civilian explosions generally preclude precise clinical histories. Second, brain injuries are usually accompanied by injuries to organs that are even more vulnerable to blast, including lung, long bones, gastrointestinal tract, and heart. Damage to these organs can produce air emboli, fat emboli, and hypoxia/ischemia that some authors have argued are more damaging to the brain than the direct effects of the blast [10]. Pathology from these causes may be hard to distinguish from that due to blast, even though embolic brain injuries usually have characteristic anatomical distributions.

Surprisingly few clinical studies have examined the effects of primary blast injuries on the brain. In some, the description is superficial and some even lack illustrations. 
Most consist of individual case reports or small series studied at autopsy or neuroradiologically. The first autopsy reports appeared in the WWI era. Several autopsy series appeared during and just after WWII, and a few small series have appeared in the past 60 years. The older reports are difficult to access, and none used the modern morphological and immunohistochemical techniques necessary to identify the changes in small, unmyelinated axons that are characteristic of diffuse axonal injury. Perhaps the most thorough clinical study is the description of the pathological changes in the brains of 37 minke whales killed immediately by a supersonic explosive grenade detonated in the thorax or abdomen [14]. Detonations within $1 \mathrm{~m}$ of the brain produced skull fractures and extensive epidural, subdural, subarachnoid, and intracerebral hemorrhages similar to the damage found after severe direct closed injury to the head. Among the changes produced by more distant explosions were deep white matter hemorrhages that were located in areas including the corpus callosum and thalamus and resembled the characteristic findings of deceleration and acceleration injury. Because the whales survived for less than 1 to $2 \mathrm{~h}$, whether these forces also produced diffuse axonal injury could not be determined.

Small or microscopic intracerebral and leptomeningeal hemorrhages are the most commonly described pathology in the human literature. In 1916, for example, F. W. Mott presented photomicrographs illustrating multiple punctate hemorrhages throughout the subcortical white matter, corpus callosum, and basal ganglia of a man who died 2 days after being rendered unconscious and buried by a shell exploding in a parapet [4]. Another contemporary report described brains subjected to aerial explosion, with hemorrhages due to rupture of small vessels in many places and punctiform hemorrhages throughout the white matter; no illustrations were provided [15]. Cohen and Biskind reviewed the files of the Armed Forces Institute of Pathology and reported the pathological findings in the brains of the only nine soldiers from WWII who died from blast injury under circumstances that were reasonably well documented [16]. All these soldiers died within 5 days of an atmospheric blast that produced no major external wounds of the head. The leptomeninges and brain were hyperemic in all cases, four brains demonstrated diffuse leptomeningeal hemorrhages, and five had intracerebral hemorrhages. The intracerebral hemorrhages were microscopic in three cases. One had an extensive bleed and laceration in the cerebellum in addition to numerous punctate bleeds in the cerebrum. In the third, multiple small hemorrhages occupied 1 to $2 \mathrm{~cm}$ of the right temporal lobe. Multifocal small hemorrhages in white matter are characteristic of closed head injuries and, when located in deep, parasagittal areas of the brain, they can be associated with diffuse axonal injury. However, this clinical material allows only the tentative conclusion that the changes are the direct effect of primary blast injuries, because multifocal petechial hemorrhages in white matter can result from secondary and tertiary injuries, carbon monoxide intoxication, and air and fat emboli.

Larger bleeds have also been observed in human specimens. For example, Cramer et al. reported, but did not illustrate, the autopsy findings in the brain of one 24-year-old American private who developed a massive intracerebral hematoma in the internal capsule, caudate nucleus, and thalamus 3 months after being rendered unconscious by a mortar shell explosion but receiving no external injury of the head or elsewhere [6]. These authors referred to two other cases in the literature of hemorrhage into a lacerated frontal lobe occurring several weeks after "cerebral blast concussion" (shell explosion) with no solid blow to the head and to additional case reports of cerebral edema, petechial and meningeal hemorrhages, cerebral contusion and laceration, and intracerebral and subdural hematoma after a cerebral blast injury. More recent single-case reports have computed axial tomography scans showing subdural hematoma [17] and epidural hematoma [18] following industrial steam explosions and an autopsy showing contrecoup contusions and lacerations of the surfaces of the frontal and temporal lobes of a 23-year-old who was fatally wounded by an underwater firecracker explosion [19].

\section{EXPERIMENTAL ANIMAL STUDIES ON BLAST- RELATED BRAIN INJURY}

The vulnerability of the brain to secondary and tertiary blast injury is clear, but whether primary blast injury has direct effects on the brain remains unresolved. However, primary blast injury has been suggested to cause TBI directly from contusion, edema, hemorrhage, and diffuse axonal damage [9]. While clinical studies have not been able to resolve the precise neuropathology of primary blast injury to the brain, some limited experimental work has been done in animal models. These few animal studies suggest that primary blast injury can damage the brain. 
Cernak and colleagues exposed rats and rabbits to primary blast, delivering the blast via a "shock tube" [20-22]. The blast was calibrated to induce a moderate level of lung damage. The animals were constrained to prevent secondary and tertiary blast injuries. Histological examination of the hippocampus and brainstem indicated that the blast exposure resulted in histological changes in the central nervous system (CNS), indicating neural injury. The authors noted expansion of perineuronal spaces, cytoplasmic vacuoles, changes in myelin structure, and axoplasmic shrinkage. These changes are consistent with diffuse axonal damage. They also noted impaired performance on an active avoidance task and biochemical changes indicative of oxidative stress.

Rats subjected to primary blast exposure in a concrete bunker were found to have widespread microglial activation in the brain, which occurs after CNS trauma [23]. The microglial activation was particularly pronounced in the superficial regions of the cerebral and cerebellar cortex. Performance on motor tasks in rats was impaired after exposure to primary blast explosions [24]. These investigators also reported degeneration of neurons in the cerebral cortex. Thus, in limited experimental studies, exposure of animals to primary blast injury suggests structural changes occur in the brain that can lead to neurological impairment.

\section{SUMMARY AND CONCLUSIONS}

The available clinical studies of blast injuries show focal damage similar to that found in other types of closed head injuries but have not determined whether diffuse axonal injury also occurs. In general, the studies are antiquated, describe the pathology superficially, and lack the precise clinical detail necessary to exclude secondary and tertiary injuries and assign the changes to the blast itself. Animal experiments using blast injury models to study primary blast injury are very limited but suggest a number of pathological changes in the brain, such as outer-layer cortical neuronal and diffuse white matter damage, including changes in myelin and axonal structure. The degree to which these animal studies can be extrapolated to humans is uncertain, especially since data are lacking for larger species and species that are structurally more similar to humans, such as nonhuman primates. Additional autopsy studies are needed to apply modern morphological methods to cases in which the cir- cumstances of the injuries are known in detail. Better understanding of the primary neuropathology will be critical in the potential design of therapeutic approaches for the treatment of primary blast injury.

\section{ACKNOWLEDGMENTS}

\section{Author Contributions:}

Drafting of manuscript: J. D. Kocsis, A. Tessler. Critical revision of manuscript for important intellectual content: J. D. Kocsis, A. Tessler.

Financial Disclosures: The authors have declared that no competing interests exist.

Funding/Support: This material was supported by the Department of Veterans Affairs.

\section{REFERENCES}

1. Wightman JM, Gladish SL. Explosions and blasts injuries. Ann Emerg Med. 2001;37(6):664-78. [PMID: 11385339] DOI:10.1067/mem.2001.114906

2. DePalma RG, Burris DG, Champion HR, Hodgson MJ. Blast injuries. N Engl J Med. 2005;352(13):1335-42.

[PMID: 15800229] DOI:10.1056/NEJMra042083

3. Murray CK, Reynolds JC, Schroeder JM, Harrison MB, Evans OM, Hospenthal DR. Spectrum of care provided at an echelon II medical unit during Operation Iraqi Freedom. Mil Med. 2005;170(6):516-20. [PMID: 16001604]

4. Mott FW. The effects of high explosives upon the central nervous system. Lancet. 1916;1:441-49.

5. Fabing HD. Cerebral blast syndrome in combat soldiers. Arch Neurol Psychiatry. 1947;57:14-57.

6. Cramer F, Paster S, Stephenson C. Cerebral injuries due to explosion waves, cerebral blast concussion; a pathological, clinical and electroencephalographic study. Arch Neurol Psychiatry. 1949;61(1):1-20. [PMID: 18108324$]$

7. Macleod AD. Shell shock, Gordon Holmes and the Great War. J R Soc Med. 2004;97(2):86-89. [PMID: 14749410] DOI:10.1258/jrsm.97.2.86

8. Povlishock JT, Katz DI. Update of neuropathology and neurological recovery after traumatic brain injury. J Head Trauma Rehabil. 2005;20(1):76-94. [PMID: 15668572] DOI:10.1097/00001199-200501000-00008

9. Guy, RJ, Glover MA, Cripps NP. Primary blast injury: Pathophysiology and implications for treatment. Part III: Injury to the central nervous system and the limbs. J R Nav Med Serv. 2000;86(1):27-31. [PMID: 10892035] 
10. Mayorga MA. The pathology of primary blast overpressure injury. Toxicology. 1997;121(1):17-28. [PMID: 9217312] DOI:10.1016/S0300-483X(97)03652-4

11. Taber KH, Warden DL, Hurley RA. Blast-related traumatic brain injury: What is known? J Neuropsychiatry Clin Neurosci. 2006;18(2):141-45. [PMID: 16720789]

12. Denny-Brown D. Cerebral concussion. Physiol Rev. 1945; 25:296-325.

13. Clemedson CJ. Blast injury. Physiol Rev. 1956;36(3):336-54. [PMID: 13359127]

14. Knudsen SK, Øen EO. Blast-induced neurotrauma in whales. Neurosci Res. 2003;46(3):377-86.

[PMID: 12804799] DOI:10.1016/S0168-0102(03)00101-9

15. Report of the War Office Committee of Enquiry into "Shellshock.” London (UK): HMSO; 1922.

16. Cohen H, Biskind GR. Pathologic aspects of atmospheric blast injuries in man. Arch Pathol. 1946;42:12-34.

17. Murthy JM, Chopra JS, Gulati DR. Subdural hematoma in an adult following a blast injury. Case report. J Neurosurg. 1979;50(2):260-61. [PMID: 430143] DOI:10.3171/jns.1979.50.2.0260

18. Yilmaz S, Pekdemir M. An unusual primary blast injury: Traumatic brain injury due to primary blast injury. Am J Emerg Med. 2007;25(1):97-98. [PMID: 17157696] DOI:10.1016/j.ajem.2006.04.014

19. Hirsch AE, Ommaya AK. Head injury caused by underwater explosion of a firecracker. Case report. J Neurosurg.
1972;37(1):95-99. [PMID: 5037050]

DOI:10.3171/jns.1972.37.1.0095

20. Cernak I, Savic J, Malicevic Z, Zunic G, Radosevic P, Ivanovic I, Davidovic L. Involvement of the central nervous system in the general response to pulmonary blast injury. J Trauma. 1996;40(3 Suppl):S100-104. [PMID: 8606388] DOI:10.1097/00005373-199603001-00023

21. Cernak I, Wang Z, Jiang J, Bian X, Savic J. Ultrastructural and functional characteristics of blast injury-induced neurotrauma. J Trauma. 2001;50(4):695-706. [PMID: 11303167] DOI:10.1097/00005373-200104000-00017

22. Cernak I, Wang Z, Jiang J, Bian X, Savic J. Cognitive deficits following blast injury-induced neurotrauma: Possible involvement of nitric oxide. Brain Inj. 2001;15(7):593-612. [PMID: 11429089] DOI:10.1080/02699050010009559

23. Kaur C, Singh J, Lim MK, Ng BL, Yap EP, Ling EA. The response of neurons and microglia to blast injury in the rat brain. Neuropathol Appl Neurobiol. 1995;21(5):369-77.

[PMID: 8632831] DOI:10.1111/j.1365-2990.1995.tb01073.x

24. Moochhala SM, Md S, Lu J, Teng CH, Greengrass C. Neuroprotective role of aminoguanidine in behavioral changes after blast injury. J Trauma. 2004;56(2):393-403.

[PMID: 14960985] DOI:10.1097/01.TA.0000066181.50879.7A

Submitted for publication August 13, 2008. Accepted in revised form April 15, 2009. 